\title{
OEBE-STARS WITH POSSIBLE COMPACT COMPANIONS OBSERVED AT X-RAYS
}

\author{
E.J.A. MEURS \\ Dublin Institute for Advanced Studies, School of Cosmic Physics, Ireland \\ and \\ A.J.M. PITERS \\ Sterrenkundig Instituut Anton Pannekoek, University of Amsterdam, The Netherlands
}

\begin{abstract}
A survey of bright O- and B-type stars has been conducted from the ROSAT All Sky Survey data, aiming at the detection of compact companions expected for Oe and Be stars. Concurrent $\mathrm{H} \alpha$ spectroscopy has been used to establish the level of activity of these stars around the time of the $\mathrm{X}$-ray observations. The detection rate of $\mathrm{OB}$ and $\mathrm{OeBe}$ stars as well as their average $X$-ray over bolometric luminosities suggest little difference between these two groups of objects. Instead of accretion onto a compact object (NS, WD), the X-ray emission from OeBe stars may be produced by stellar winds as has been proposed for $\mathrm{O}$ - and early B-type stars in general. Flexibilities in the available models for $\mathrm{X}$-ray emission from accreting WDs may allow the X-ray emission from such objects to remain below a detectable level. These investigations are now being extended to pointed observations in the ROSAT Archive.
\end{abstract}

Key words: stars: OeBe - white dwarfs - neutron stars - accretion - X-rays

\section{The case for white dwarf companions to OeBe stars}

Evolutionary calculations regarding the stellar population in our Galaxy have been quite successful in predicting the total number of evolved binaries of a particular type that should be around at the present epoch. Meurs \& van den Heuvel (1989) derived, e.g., the expected number of persistent Xray binaries which compares favourably with an estimated total number of these objects from the available observational data. The implications of the concurrent calculation of the likely number of Wolf-Rayet stars produced via close binary evolution are described in Meurs \& van den Heuvel (these proceedings). The role of close binary evolution in the context of Galactic stellar populations was treated more comprehensively by Pols (1993) and one of the results concerns compact companions to OeBe stars.

The OeBe stars exhibit high rotational velocities that may find a natural explanation from the angular momentum of material transferred between the two components in a close binary system. If the $\mathrm{OeBe}$ stars indeed went through a stage of mass transfer, then the $\mathrm{O}$ or $\mathrm{B}$ star is a rejuvenated secondary while the original primary should now be a helium star, white dwarf, neutron star or black hole. The cause for the emission line characteristics present in about $10 \%$ of all (O)B stars - is not very well known, but various observational studies indicate that these stars lose mass in an equatorial 
wind (see for an overview and references Slettebak 1988). If the companion is a neutron star, it will accrete material and emit X-rays during each passage through the equatorial excretion disk. In this situation the (new) primary can feed the NS while staying well within its Roche lobe.

This provides an attractive scenario for the $\mathrm{Be} / \mathrm{X}$-ray binaries, of which several are known (van den Heuvel \& Rappaport 1987). They have luminosities $L_{X} \approx 10^{34}-10^{38} \mathrm{erg} \mathrm{s}^{-1}$ and are found among the early-Be (and Oe) stars, which is understandable in view of the high masses needed for NS production. More towards intermediate masses, the later Be stars, the companion is rather a WD but could still be accreting material from the equatorial wind. On the basis of a simple wind model Waters et al. (1989) estimate luminosities of the order of $L_{X} \approx 10^{29}-10^{33} \mathrm{erg} \mathrm{s}^{-1}$ for these systems. This is lower than for the NS companions, but would still be detectable for many nearer (Oe)Be stars. With detailed evolutionary calculations Pols et al. (1991) expect among the B stars in the Bright Star Catalogue (BSC) about $4 \mathrm{Be}+\mathrm{NS}$ systems, about $20 \mathrm{Be}+\mathrm{WD}$ systems and about $60 \mathrm{Be}+\mathrm{He}-$ star systems. Although the X-ray luminosities go down for WD companions, they are more numerous than the NS companions (the stellar mass function is very steep).

So far, about five of the later Be stars have been detected at X-rays and an accreting WD companion has been suggested for these (Waters 1989). Since the X-ray spectra of such systems could be rather soft (e.g., van der Woerd 1987), it seemed worthwile to inspect the ROSAT All-Sky Survey for detections of $\mathrm{O}(\mathrm{e}) \mathrm{B}(\mathrm{e})$ stars in the BSC.

\section{X-ray and optical data}

The ROSAT satellite scanned all of the sky with the PSPC detector, giving out eventually a source list within strips of $2^{\circ}$ wide. The PSPC window spans 0.1-2.4 keV and was divided in bands $0.1-0.4,0.4-1.0$, and $1.0-2.4 \mathrm{keV}$ in order to determine hardness ratios. For the present results we used $75 \%$ of the all-sky data and took care in applying additional position and vignetting corrections where required (Meurs et al. 1992). The associations with the $\mathrm{O}(\mathrm{e}) \mathrm{B}(\mathrm{e})$ stars in the $\mathrm{BSC}$ were obtained on the basis of positional coincidence. The emission character of the 0 stars was established as described in Meurs et al. (1992, section 3).

The stars scanned in the Survey were also observed at other wavelengths, as simultaneously with the $\mathrm{X}$-ray observations as possible (correlations with $L_{X}$ predicted in the simple wind model). Of the various additional observations (see Meurs et al. 1992) we refer here mainly to the high-resolution $\mathrm{H} \alpha$ spectra that we examined for the presence of the characteristic emission lines at the time of the $\mathrm{X}$-ray measurement. In total we find detections for 10 OeBe stars from the BSC, while 9 detected stars did have $\mathrm{H} \alpha$ in emission 
during the ROSAT scanning.

It is somewhat surprising that the detected OeBe stars are not recognizeably different from normal $O B$ stars and that none of the previously suggested WD-companion candidates was detected with ROSAT. The detection rates for $\mathrm{OB}$ and $\mathrm{OeBe}$ stars do not differ significantly. The greatest difference between these rates is obtained using our concurrent $\mathrm{H} \alpha$-emission/ absorption data, but gives only a difference of just over $2 \sigma$. However, the stars with $\mathrm{H} \alpha$ in emission exhibit the lower rate, contrary to what was anticipated. Also the ratio of $X$-ray to bolometric luminosity is comparable for the two groups of objects (emission and non-emission) - as far as this can be established in the presence of the ROSAT detection limit (see Meurs et al. 1992, fig.1 and section 3). Further, where enough counts were collected to consider hardness ratios, these were not suggesting much difference either (see fig. 2 in Meurs et al. ), except for a few very soft $\beta$-Cep-stars and hardest spectra for two Be+NS cases (X Per and $\pi$ Aqr). As to the individual non-detected $\mathrm{Be}+\mathrm{WD}$ candidates, the case of $\gamma$ Cas is a bit undefined. It is probably detected in the Survey but is in the part of the sky not covered by our present material. Waters (1989) suggests that $\gamma$ Cas is a Be star with a NS in a wide orbit. On the basis of a pointed ROSAT PSPC observation, Haberl (in preparation) argues for a $\mathrm{Be}+\mathrm{WD}$ system. The hardness ratios invoked here leave this an uncertain interpretation, however (see below).

\section{Where are the WDs?}

The X-ray emission from the OeBe stars is thus not obviously different from that of $\mathrm{OB}$ stars in general. This means, for the $\mathrm{O}(\mathrm{e})$ and early $\mathrm{B}(\mathrm{e})$ stars, that the $\mathrm{X}$-ray emission is likely to originate from shocks in radiatively driven winds (Lucy 1982). This is supported by the roughly constant $L_{X} / L_{b o l}$ ratio and by the rapid decrease in detection rate around types B2/B3 when the stellar winds are expected to become too weak to generate $\mathrm{X}$-rays (see fig. 3 in Meurs et al. 1992). For later-B(e) types the situation is less clear. One conceivable possibility is that these stars have late-type companions with coronae. The X-ray luminosities of chromospherically active stars (of types F, G, K) are compatible with the luminosities found for the later B stars. Two of the detections (one Be, one normal B) are in fact known to possess such companions. Another possibility for the later B stars would be shocks in a weak wind that is enhanced by rotation (Marlborough 1987), but we do not find any correlation of their X-ray emission with $v \sin i$.

This seems to leave little space for any accreting WD companion. These should exist in substantial numbers, however (unless there are still major surprises concerning the evolution of less massive binaries). One can think of several possibilities for the WD companions not to be visible in the ROSAT Survey data. The predictions of Waters et al. (1989) are based on a wind 
model that may have been derived with too simplistic an extrapolation from heavier systems. On the other hand, a considerable uncertainty is attached to the X-ray spectra of accreting WDs ( $c f$. van der Woerd 1987). It could be that low accretion rates rather lead to hard spectra (which then are easily missed by ROSAT), whereas high accretion rates produce very soft spectra that may suffer from heavy absorption and reprocessing in the disk (see Apparao 1991).

Some further possibilities are: not all post-mass transfer B stars show up as Be star or only episodically (more specifically, the WD star may not be always near the densest equatorial outstream); are the B+WD systems (also) among 'normal' OB stars; are there single Be stars; are the Be stars in interacting binaries with accretion disks (Harmanec 1987)?

\section{A ROSAT Archive attempt}

The analysis of the ROSAT Survey data for $\mathrm{O}(\mathrm{e}) \mathrm{B}(\mathrm{e})$ stars points to several sequel studies for this sample. One useful project is to look into the ROSAT Archive for any observations in which one of the non-detected Be+WD candidates may appear. Unfortunately, the five or so cases (see above) are not in any field and - and excluding the three cases that may have a NS companion in a wide orbit (like $\gamma$ Cas) - only HR 4009, which may have a NS companion in a wide orbit, is included in an exposure (a PSPC field, in which the position of HR 4009 is $16^{\prime} .5$ from the centre of the image). Inspection shows two weak nearby sources, which are not likely associated; an accurate calibration of the positions in this field will be attempted. A further way to find out more about these WD candidates would be a detailed analysis of the Survey's Photon Event Tables, which has not been fully exploited yet.

\section{References}

Apparao, K.M.V. 1991, $A \mathcal{G} A$ 248, 139

Harmanec, P. 1987, in: A. Slettebak \& T.P. Snow (eds.), Physics of Be Stars (Cambridge: CUP), p. 339

van den Heuvel, E.P.J., Rappaport, S. 1987, in: A. Slettebak \& T.P. Snow (eds.), Physics of Be Stars (Cambridge: CUP), p. 291

Marlborough, J.M. 1987, in: A. Slettebak \& T.P. Snow (eds.), Physics of Be Stars (Cambridge: CUP), p. 316

Meurs, E.J.A., van den Heuvel, E.P.J. 1989, $A \& A$ 226, 88

Meurs, E.J.A., Piters, A.J.M., Pols, O.R. et al. 1992, A\&A (Letters) 265, L41

Pols, O.R., Cote, J., Waters, L.B.F.M., Heise, J. 1991, $A \& A$ 241, 419

Pols, O.R. 1993, PhD thesis, University of Amsterdam

Slettebak, A. 1988, PASP 100, 770

Waters, L.B.F.M. 1989, Proc. 23rd ESLAB Symp., ESA SP-296 1, 25

Waters, L.B.F.M., Pols, O.R., Hogeveen, S.J., Cote, J., van den Heuvel, E.P.J. 1989, $A \mathscr{E} A$ (Letters) 220, L1

van der Woerd, H.J. 1987, PhD thesis, University of Amsterdam 


\section{DISCUSSION:}

Maeder: A basic problem in stellar evolution is the relation between initial masses and final compact objects. Classically, there is a well defined correspondence of the mass ranges. But, as a result of so many processes, the situation may well be much more complicated. We already know that neutron stars may come out of massive stars and some of them could even lead to white dwarfs in cases of extreme mass loss. Thus, such searches are quite important.

Meurs: Any conclusion about possible masses for White Dwarfs and about correspondences of mass ranges - on the basis of the present project - should however await further developed knowledge regarding the conditions governing the observability of the White Dwarf companions: the distribution of any accreting material, orbital distributions, emerging WD Xray spectra, etc.

Lindsey Smith: The same naive question in another form - accreting WDs become novae. So far as I am aware, novae do not occur in this kind of system. Does it not follow that $\mathrm{OeBe}+\mathrm{cc}$ do not exist?

van Kerkwijk: We know $\mathrm{OeBe}+$ n.s. systems exist; these are the $\mathrm{Be} / \mathrm{X}$-ray binaries, and hence it seems hard to imagine that no $\mathrm{OeBe}+\mathrm{WD}$ systems would form. The question whether they should be observable as novae, mainly depends on whether they can accrete enough matter - at the right rate - during their lifetime. The accretion rate is probably about $10^{-9} \mathrm{M} \odot \mathrm{yr}^{-1}$ for the $10-50 \%$ "on-time" of the total Be-star lifetime (a couple of million years).

Gies: Do you include possible asymmetric supernova explosions in your calculations of $\mathrm{Be}+$ n.s. systems? These can give a substantial kick velocity to the newborn neutron star, and can contribute to break up of the binary system.

Meurs: The possible break-up as a consequence of kick velocities has been taken into account in a statistical sense.

Hill: Just a comment: if some Be stars have disks inclined to the white dwarf orbital plane you would not see steady X-ray emission but rather bursts as the WD passed through the disk. Such systems could be missed.

van Kerkwijk: In the evolutionary scenario that we are testing, one expects that the rotational and orbital axes are aligned, since the rapid rotation of the $\mathrm{Be}$ star is most likely a result from mass transfer in the preceeding evolution. Since no violent events occurred like the supernova in a more massive system (which forms a Be/X-ray binary), the White dwarf should therefore orbit in the equatorial plane of the Be star. 